\title{
A Link among Human Gut Microbiome and COVID-19?
}

\section{Kawalpreet K Aneja*}

Biology Educator for Randolph Career and Technical Institute, Henry Ave, Philadelphia, USA

*Corresponding Author: Kawalpreet K Aneja, Biology Educator for Randolph Career and Technical Institute, Henry Ave, Philadelphia, USA.
Received: March 29, 2021

Published: April 30, 2021

(C) All rights are reserved by Kawalpreet $\mathbf{K}$

Aneja.

\section{Abstract}

The microbiome is composed of bacteria, archaea, fungi, protists, and viruses. The human microbiome is well known but still unfolding in various sites like skin, saliva, mucus, placenta, seminal and ovarian fluids, respiratory, gastrointestinal, and urogenital tract. The mucus lining of our body plays a major role in first-line defense against pathogens. Dietary fiber or plant-based diet and microbial foods strengthen our mucus lining and modulate the innate, adaptive, and regulatory immune system. Perhaps probiotics or microbial foods, personalized nutrition, and supplementation to support gut microbiota should be part of a natural or prescribed regimen to fight against COVID-19 like diseases. Recent studies have found a link between COVID-19 and perturbed gut microbiota. The reduced immunomodulatory bacteria like Faecalibacterium prausnitzii, Eubacterium rectale, and Bifidobacteria were concordant with a surge in inflammatory cytokines like CXCL10, CCL2, IL-10 and TNF $\alpha$ and blood markers such as C reactive protein, lactate dehydrogenase, aspartate aminotransferase, and gamma-glutamyl transferase in patients up to 30 days after COVID-19 resolution. Strong and healthy gut microbiota provide multiple defense systems such as a thick mucus layer that promotes the growth of commensal bacteria, intestinal epithelial cells with tight junctions, and regulate various host factors like antimicrobial peptides, immunoglobulin A, proteases, etc. Can we stop COVID-19 like diseases by improving gut microbiota?

Keywords: COVID-19; Cytokine Storm; Gut Microbiota; Gastrointestinal Tract; Mucin; Short-Chain Fatty Acids

\section{Abbreviations}

GI: Gastrointestinal; GIM: Gastrointestinal Microbiota; FOS: Fructo-oligosaccharides; GOS: Galacto-oligosaccharides; BDNF: Brainderived Neurotrophic Factor; Trp: Tryptophan; GBM: State-of the-Science-Gut-Brain-Bidirectinal Axis; 5HT: Serotonin; ACE2: Angiotensin-converting Enzyme 2; CD: Crohn's Disease; T1D: Type1 Diabetes; COVID-19: Coronavirus Disease 2019

\section{Introduction}

All living organisms are exposed to metagenome or microbiota. The human gastrointestinal tract (GI) contains all three domains of life: bacteria, archaea and eukarya. But diversity at the division or phylum level is among the lowest [1]. The most common bac- teria that contribute up to $30 \%$ of the gut and the outer intestinal mucus layer of the gut belong to phylum Cytophaga-Flavobacterium-Bacteroides or Bacteroidetes (CFB), genus Bacteroides [2,3]; phylum Firmicutes including genus Clostridium and Eubacterium; phylum Proteobacteria; phylum Actinobacteria and phylum Verrumicrobia [1,4]. Accompanied with bacteriome our GI tract also contains the human virome, fungal microbiome or mycobiome, bacteriophagome, or predators of the bacterial microbiome [5]. Gastrointestinal microbiota (GIM) has been named as a virtual metabolic organ because they directly or indirectly regulate various metabolic pathways of carbohydrates, lipids, proteins, and nucleotides and navigate energy homeostasis processes via these pathways [4]. Through the soap opera of these pathways, GIM

Citation: Kawalpreet K Aneja. "A Link among Human Gut Microbiome and COVID-19?". Acta Scientific Microbiology 4.5 (2021): 134-141. 
helps in adsorption and absorption of various metabolites, production of short-chain fatty acids [SCFAs], amines, phenols/indoles, and sulfurous compounds, vitamin $\mathrm{B}$ and vitamin $\mathrm{K}$ synthesis, and the metabolism of bile acids [4]. The prebiotic inulin influences the production of metabolic hormones such as glucagon-like peptide-1, peptide YY, ghrelin, and leptin [6]. Other prebiotics like fructo-oligosaccharides [FOS] and galacto-oligosaccharides (GOS) increase BDNF, serotonin, GABAb receptors levels while reducing cortisone and L-Trp which are known culprits for anti-anxiety and anti-depressant effects [7]. State-of-the-Science-Gut-Brain-Bidirectional Axis (GBM) is highly manipulated by gut microbiota. GIM controls GBM by neurochemical, neuroendocrinal, and neuroimmunal mechanisms. Lactobacillus sps, Candida, Streptococcus, E. coli, and Enterococcus can synthesize serotonin (5HT) in the gut which affects sleep, appetite, moods, and cognition [8]. Clostridiales regulate the formation and release of tryptophan, an essential precursor to serotonin [9]. Microbial metabolites like short-chain fatty acids, bile acids, and tryptophan can communicate between gut and brain directly or through vagal/spinal highways. Since GIM releases these humoral agents and distally or proximally affects other glands, organs, and receptors, GIM is known as an endocrine organ. GIM has been known to change dietary lipid phophatidylcholine (PC) into choline to betaine and then to trimethylamine/trimethylamine$\mathrm{N}$-oxide which increases the risk of atherosclerosis. This complex symbiotic relationship of the human microbiome is still far to understand because until now there is only one highly evolutionary conserved 16srRNA sequencing method available to characterize different types of bacteria from Earth to Mars. Our mini-review is an effort to investigate recently documented studies showing mutualism between gut microbiota and the human immune system. How can we use this information to manipulate gut microbiota to ameliorate symptoms of COVID-19?

\section{How does our gut protect us?}

From oral cavity to colon, our GI tract is lined by a special mucus layer which is made up of a miracle protein known as mucin. The gel-forming 0-glycosylated mucin protein (MUC2) is the main protein inside the intestinal mucus lining [10] which regulates the balance of commensal vs pathogenic bacteria; the penetration of pathogenic bacteria inside various types of intestinal epithelial cells, and also MUC2 incites the immune response against pathogens. From various experimental studies, it seems that our GI tract responds to changes in diet, exercise, stress, environment, hormonal changes, even jet lag, and our moods mainly through the balance of mucus layer and a variety of commensal versus pathogenic microcosm. There are two mucus layers present above the stomach and large intestinal (Figure 1A) epithelial cells but there is only one mucus layer above the small intestine. Small intestinal epithelial lining contains a large number of Paneth cells that are known to synthesize antimicrobial peptides (AMPs) and the regenerating islet-derived 3 (Reg3) family of proteins. AMPs include a family of defensins and cathelicidins. Defensins are of three types: alpha, beta, and theta. Defensins protect against Gram-positive, Gram-negative bacteria, as well as fungi, protozoa, and viruses [11], alpha-defensins have been shown to protect against Salmonella typhimurium [12]. Reg3 is active against gram-positive bacteria [13]. Well, the large intestine is covered by two mucus layersthe inner mucus layer is made up of mainly Muc2-mucin along with Fc Ig binding protein Fcgbp or mucous cross-linker- Clca3, Zg16, Agr2, immunoglobulins, and many more proteins [14] (Figure 1B). MUC2-mucin is mainly secreted by goblet cells but regulated by the host response to microbiota and/or by microbial metabolites like SCFAs, Th2 cytokines like IL-5, and IL-13 [12]. Polymerized MUC2mucin plays a major role in controlling penetrance of commensal bacteria and holds a tough battle between commensal and pathogenic bacteria. The polymerized MUC2-mucin is the main component of the inner mucus layer. Host cells and commensal bacteria catabolize the MUC2-mucin and change it into the outer mucus layer which is habitable for the growth of commensal microbiota. The commensal microbiota regulates digestion, absorption, motility, cell proliferation, cell regeneration, hormonal balance, and the enteric nervous system. Dietary fiber is extremely important to keep commensal bacterial growth, they convert dietary fiber into shortchain fatty acids that favor bacterial growth, Muc2-mucin formation as well as helps in the synthesis of vitamin B and $\mathrm{K}$ (Figure 1B). Short-chain fatty acids and ATP also trigger an innate immune response. The mucus layer, cell junctions, transmembrane mucins, glycocalyx make the physical barrier while AMPs, the regenerating islet-derived 3 [Reg3] family of proteins, alpha, beta, and theta-defensins comprise the chemical barriers of the GI tract. The large intestine also contains a highly glycosylated Glycosylphosphatidylinositol (GPI)-anchored protein called Ly6/Plaur domaincontaining 8 (Lypd8); Lypd8 is anchored to the intestinal epithelial cells and is constitutively shed into the lumen to inhibit invasion by bacteria like Escherichia, Proteus, and Helicobacter [12] (Figure 1B). Plant-based diet or diet rich in fiber, pre-and probiotics have been proven to strengthen the mucus lining, the first innate defense against pathogens like COVID-19. 


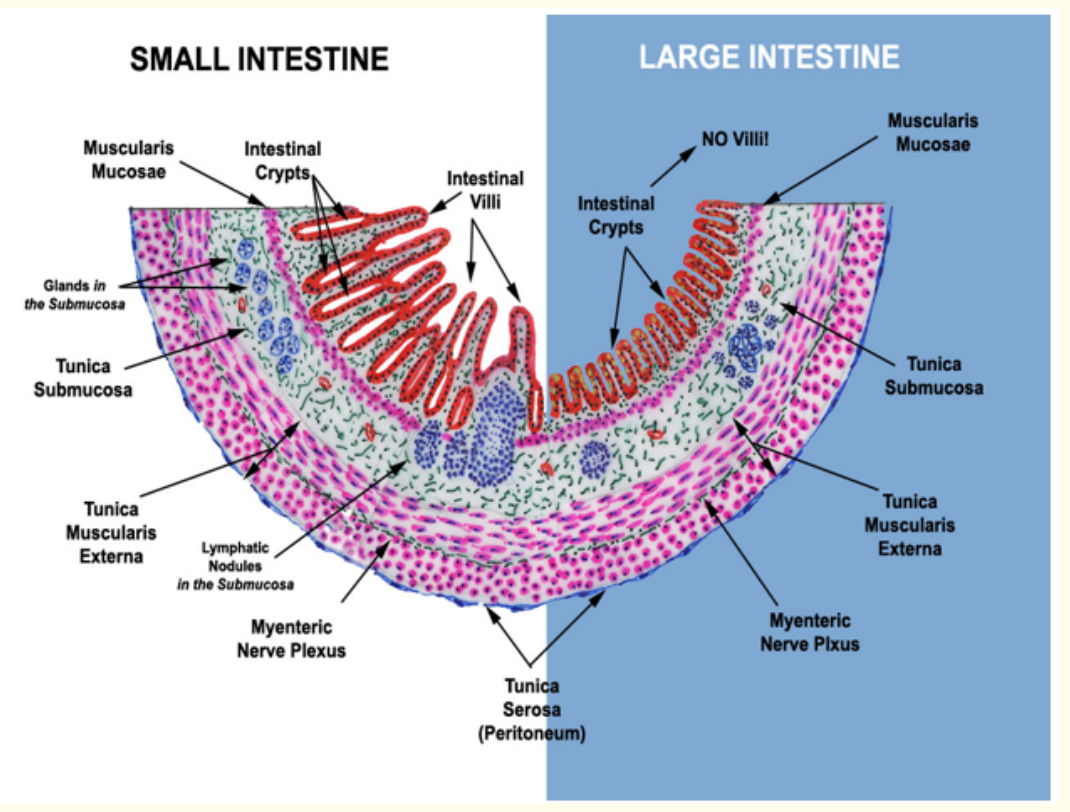

Figure 1A: The key distinguishing features of small and large intestine. Since the small intestine is mainly responsible for the absorption of nutrients, to facilitate this job it is lined by intestinal villi. The large intestine absorbs water, important nutrients like salts, electrolytes, and vitamins, synthesizes vitamin $\mathrm{K}$ and vitamin $\mathrm{B}$, and fecal bolus dries out here, villi are absent. Also, the small intestine is lined by a single mucus layer, it is full of antimicrobial peptides and Reg3 family of proteins synthesized by Paneth cells. The large intestine is lined by a double mucus layer; the inner mucus layer contains Lypd8. Figures courtesy of Dr. Thomas Caceci retired faculty, Virginia-Maryland College of Veterinary Medicine, Blacksburg, VA http://www.doctorc.net/.

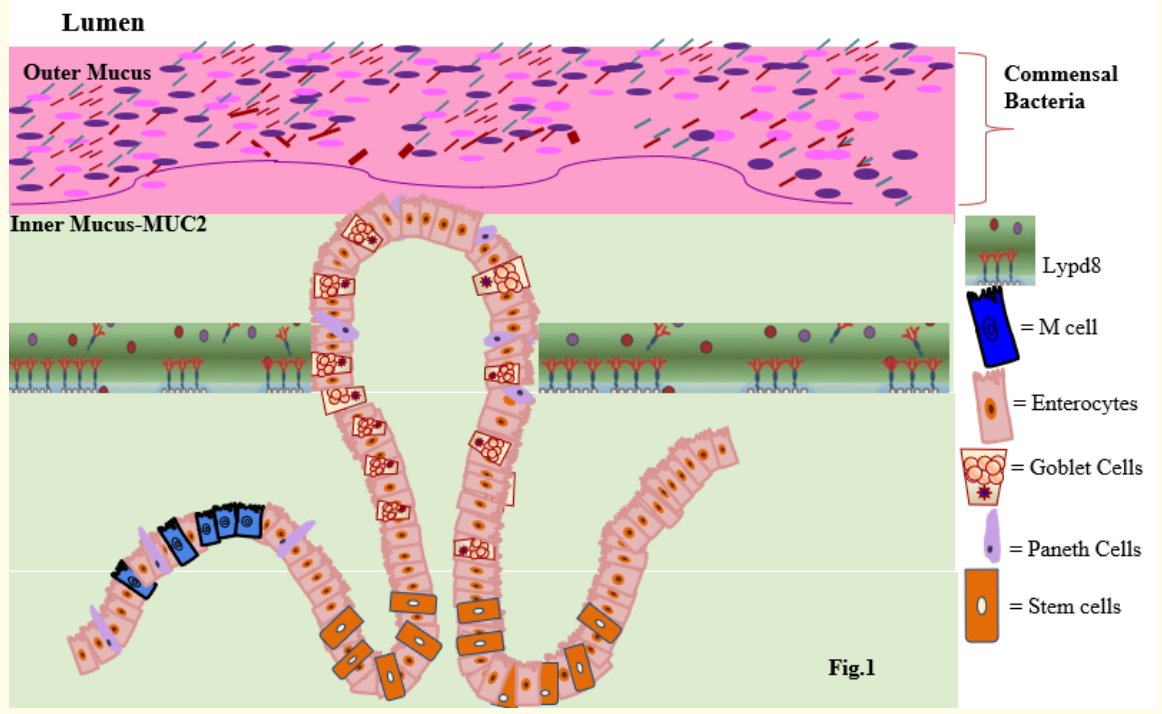

Figure 1B: Two Mucus Layers or Barriers of Large Intestine (LI). The outer mucus layer is the favorite niche of commensal bacteria and is fed by major protein or MUC- 2 of the inner mucus layer. Commensal bacteria like Bacteroides, Clostridia, Bifidobacteria produce shortchain fatty acids from dietary fiber that regulates mucin formation, the first-line defense against pathogens. The inner mucus layer is also lined by Lypd8 protein, a barrier for the entrance of commensal or pathogenic bacteria inside the enterocytes. The commensal bacteria like Faecalibacterium prausnitzii, Eubacterium rectale, and Bifidobacteria are highly reduced in COVID-19 patients. This figure was made with help of Okumura and Takeda 2017, Fujimoto and Uematsu 2020, Cummings and Macfarlane 1991, Markowiak-Kopeć and Śliżewska 2020, Yeoh., et al. 2020. 
Indestructible COVID-19 and the global carnage (plague)

Coronaviruses are enveloped, non-segmented viruses, which means that the whole genome is on a single positive-sense RNA strand as compare to segmented viruses which contain genome in small fragments; the viral genome is in the size from 26 to $32 \mathrm{ki}$ lobases, the largest known viral RNA genome [15] and COVID-19 genome is approximately $30 \mathrm{~kb}(29,811$ nucleotides $)$ in length with a $5^{\prime}$-cap structure and $3^{\prime}$-poly-A tail.

\section{COVID-19 and immune response}

COVID-19 is recognized by pattern recognition receptors of extracellular and endosomal Toll-like receptors [TLRs], TLR3 and TLR 7/8, and the cytosolic RNA sensor, retinoic acid-inducible RIGI like receptors (RLRs) (Figure 2). The TLRs and RLRS belong to the first line pattern recognition receptor category. We will perhaps find the involvement of nucleotide-binding and oligomerization domain-like receptors (NLRs), C-type lectin-like receptors (CLRs) and cytoplasmic DNA receptors shortly. These first-line receptors instigate a cascade of signaling events that involve MYD88, TRIF, IRAKS, TRAFs, transcription factors like, nuclear factor $-\kappa \mathrm{B}(\mathrm{NF} \kappa \mathrm{B})$ and IFN-regulatory factor 3 (IRF3), IRF7, and are dominated by type I interferon response and IFN-stimulatory genes (ISGs) [16] (Figure 2). After Interferon is expressed; it is translocated across the cell membrane; it can signal in an autocrine or a paracrine way via the interferon- $\alpha / \beta$ receptor consisting of two subunits, IFNAR1(IFNAR; IFN receptor) and IFNAR2, this leads to phosphorylation of STAT transcription factors through the JAK-STAT pathway. STAT1 and STAT2 now heterodimerize and translocate to the nucleus to activate a broad range of ISGs (Figure 2). Second-line category receptors comprise nucleic acid receptors with direct antiviral activity, for example, double-stranded RNA (dsRNA)-activated protein kinase R (PKR; also known as eIF2AK2), 2'5'oligoadenylate synthetase 1 (OAS1), adenosine deaminase acting on RNA 1 (ADAR1) [16], PKR, OAS1, and ADAR1 are also known as ISGs. The major difference between first-line and second-line nucleic acid receptors is that the second-line receptors do not induce an immune response by transcription factors or cytokines but directly destroy viral RNA by cleavage, modification, or translational inhibition [16,17]. Different TLRs responses lead to different outcomes, for example TLR7 and TLR9 on plasmacytoid dendritic cells lead to type I IFN production while TLR8 on myeloid cells release IL-12 [16] (Figure 2). Overall excitement of innate immune response causes a storm of cytokines, chemokines, antiviral proteins that directly or indirectly, with or without adaptive immune response, cause the end of the viral realm by preventing viral replication, transcription, translation, virus assembly which ultimately leads to cell death including apoptosis, necroptosis, and pyroptosis.

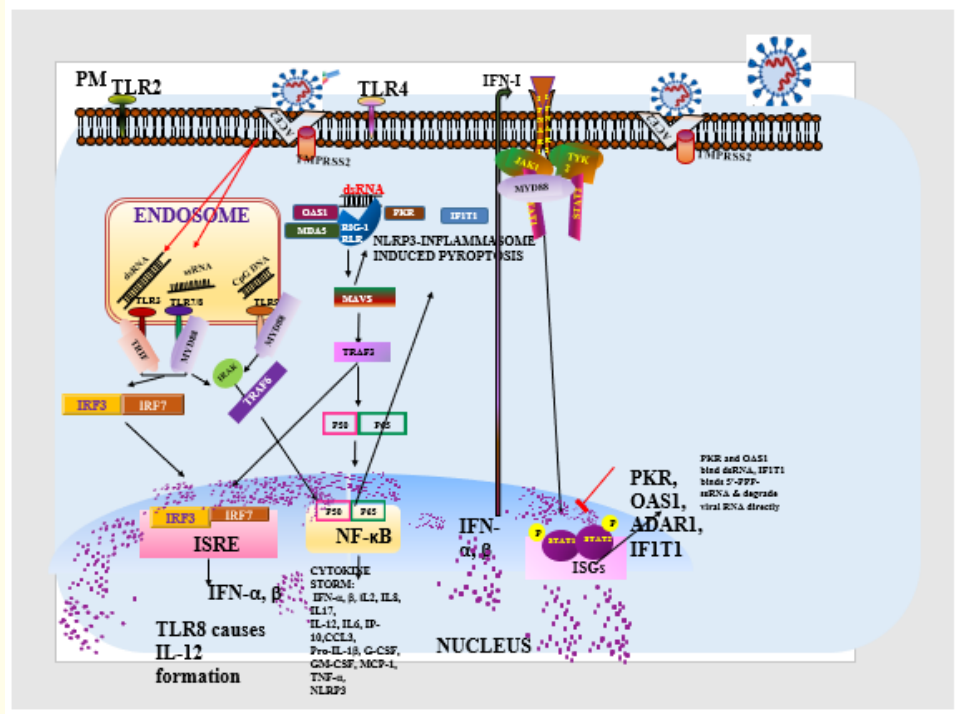

Figure 2: Innate immune response and COVID-19: Viruses activate innate immune response by pattern recognition receptors (PRRs) like Toll-like receptors, (TLRs) RIG-like receptors (RLRs), NOD-like receptors (NLRs), and C-type lectin receptors (CLRs), only TLRs and RLRs that are used by COVID-19 are shown above. Endosomal TLR3s recognize double-stranded RNA, Poly-IC, viral RNA, siRNA, and endogenous mRNA; TLR7 and 8 recognize single-stranded RNA; TLR9 binds to CpG-ODN. TLRs activate the signal pathway that starts from adaptor proteins like TRIF/MyD88 then through IRAK-TRAF6 to transcription factors like IRF3/IRF7/NF-KB/AP-1 which translocate to nucleus and cause type I IFN and subsequent ISGs expression. Secreted type I IFNs can translocate across the cell membrane in an autocrine or a paracrine fashion through IFN- $\alpha$ / $\beta$ receptor, this activates the phosphorylation of STAT1 and STAT2 via JAK family kinases, JAK1 and TYK2. Phosphorylated STATs heterodimerize and translocate to the nucleus to activate a broad range expression of ISGs. Besides TLRs, RIG-1 and MDA5 detect dsRNA in the cytosol and activate MAVS to induce type I IFN production via IRF3-IRF7; MDA5 also activates apoptosis and signals NLRP3 inflammasome formation which causes pyroptosis. The second line receptors PKR, OAS, IF1T1directly destroy viral RNA by cleavage, modification, or translational inhibition. Inflammatory cytokine storm is the hallmark of COVID-19 which leads to plasma leakage, vascular permeability, and disseminated vascular coagulation, and uncontrolled inflammatory response that account for life-threatening respiratory symptoms. This figure was adapted from Aneja., et al. 2020, Krishnan., et al. 2007, Schuster., et al. 2019, Schlee and Gunther 2016, Huang., et al. 2019, and Muus., et al. 2020. 


\section{Cytokine storm}

Inflammatory cytokine storm is the hallmark of COVID-19 which leads to plasma leakage, vascular permeability, and disseminated vascular coagulation, and uncontrolled inflammatory response that account for life-threatening respiratory symptoms [18]. The plasma concentrations of IL-1 $\beta$, IL-1ra, IL-7, IL-8, IL-9, IL-10, basic FGF, G-CSF, GM-CSF, IFN- $\gamma$, IP-10, MCP-1, MIP- $1 \alpha$, MIP-1 $\beta$, PDGF, TNF $\alpha$, and VEGF were higher in both ICU [intensive care unit] patients and non-ICU patients than in healthy adults. Moreover, when comparing ICU and non-ICU patients, plasma concentrations of IL2, IL-7, IL-10, G-CSF, IP-10, MCP-1, MIP-1 $\alpha$, and TNF $\alpha$ were higher in ICU patients than non-ICU patients, evidence that the cytokine storm is correlated with disease severity [19] (Figure 2). Selected drugs like tocilizumab-sarilumab, baricitinib-fedratinib, and fingolimod are in consideration to manage this storm [18].

\section{COVID-19 and ACE2}

The gut microbiome is just another immunological organ of our body. How does gut microbiota modulate COVID-19 invasion? COVID-19 patients manifest GI tract disturbances along with respiratory symptoms. Some patients with the COVID-19 virus in the GI tract do not have GI symptoms [20]. Patients with a low-fiber or plant-based diet have a high risk of IBD, diabetes, hypertension, obesity, and hence COVID-19 invasion. Since COVID-19 blocks angiotensin-converting enzyme 2 (ACE2), this altered function of ACE2 in the lungs leads to acute lung injury. Also, ACE2 is an important regulator of dietary amino acid homeostasis, innate immunity, gut microbial ecology, and susceptibility to colitis; therefore, its inhibition can cause intestinal inflammation. Since ACE2 regulates vital processes such as normal cardiac function, optimal beta-cell function, and insulin sensitivity [22], if ACE2 functions are blocked by COVID-19, various complications may occur. Leaky gut can increase binding of COVID-19 to ACE2 and worsen various GI symptoms, and cause spillover of COVID-19 into circulation and infect almost all internal organs expressing ACE2 [23].

One of the biggest questions is can we find the effect of different diets, probiotics, or prebiotics on mitigation of COVID-19 by priming immune response.

\section{COVID-19 and the gut microbiome}

Can we find a link between COVID-19 and the gut microbiome? The most recent evidence came from Yeoh., et al. 2020 studies which showed that several gut immunomodulatory potential commensals such as Faecalibacterium prausnitzii, Eubacterium rectale, and Bifidobacteria were underrepresented in patients and remained low in samples collected up to 30 days after COVID-19 resolution. On the other hand, patients showed an increase in Ruminococcus gnavus, Ruminococcus torques, Bacteroides dorei and Bacteroides vulgatus which is relevant with COVID19 caused immune dysregulation (Figure 1B). There are already reports about $R$. gnavus and $R$. torques co-occurring with inflammatory bowel disease $[25,26]$. B. dorei and B. vulgatus are implicated in several inflammatory gut diseases such as irritable bowel disease and ulcerative colitis [27]. This frightening changed composition of gut bacteria in COVID patients exhibited a similar pace with COVID severity causing inflammatory cytokines like CXCL10, CCL2, IL-10, and TNF- $\alpha$ and blood markers such as $\mathrm{C}$ reactive protein (CRP), lactate dehydrogenase ( $\mathrm{LDH}$ ), aspartate aminotransferase (AST) and gamma-glutamyl transferase (GGT) and erythrocyte sedimentation rate. This association between gut microbiota composition, levels of cytokines, and inflammatory markers in patients with COVID-19 strongly suggest that the gut microbiome is involved in the magnitude of COVID-19 severity via modulating host immune responses. Furthermore, the gut microbiota dysbiosis after disease resolution may be contributing to persistent symptoms [24]. How do differences in composition and function of GIM communities may contribute to inter-individual variation in cytokine responses to microbial stimulations and immune response in healthy humans' vs COVID-19 patients is a big conundrum.

\section{Inferences from other examples}

Perhaps we need to look at other examples. From host-microbial interactions, TNF $\alpha$ and IFN $\gamma$ production was found to be specifically associated with microbial metabolic pathways of palmitoleic acid metabolism and tryptophan degradation to tryptophol [28]. Gut microbiota has been known to assess and modulate both innate and adaptive immune responses. One of the best examples is dietary fiber conversion to short-chain fatty acids [SCFAs] by commensal bacteria. SCFAs are not only the energy source but also regulate cytokine production and induce expansion of regulatory $\mathrm{T}$ cells or Tregs [29,30]. A Human-derived mix of 17 Clostridia strains makes SCFAs and have anti-inflammatory properties [31,32]. Clostridia are also beneficial for epithelial permeability to food antigens to protect against allergen sensitization [33]. Crohn's disease, type 1 and type 2 diabetes, and obesity, rheumatoid arthritis clearly show dysbiosis, and symptoms of these diseases ameliorate by gut beneficial 
microbiota. An axis defined by an increased abundance of bacteria like Enterobacteriaceae, Pasteurellacaea, Veillonellaceae, and Fusobacteriaceae, and decreased abundance in Erysipelotrichales, Bacteroidales, and Clostridiales correlates strongly with inflammatory bowel disease (IBD) status [34]. Microbiome comparison between CD patients with and without antibiotic exposure indicates that antibiotic use amplifies the microbial dysbiosis associated with CD. We can diagnose $\mathrm{CD}$ at an early stage by just comparing the microbial signatures of the ileum, rectum, and fecal samples [34]. Studies in children with genetic risk factors for T1D and analysis of the microbiome in rodent models have showed associations between an altered microbiome composition potentially favoring a pro-inflammatory intestinal metabolic milieu and T1D [35]. We already know from comparison studies between antibiotic-treated versus free commensal bacteria in mice that show increased sensitization to food allergens. By selectively colonizing gnotobiotic mice, the allergy-protective capacity is conferred by a Clostridia-containing microbiota. Microarray analysis of intestinal epithelial cells from gnotobiotic mice revealed a previously unidentified mechanism by which Clostridia regulate innate lymphoid cell function and intestinal epithelial permeability to protect against allergen sensitization. These findings suggest the development of novel approaches to prevent or treat food allergy based on modulating the composition of the intestinal microbiota [33].

From above few examples of GIM modulation of innate and adaptive immune response not only against pathogens, clinical conditions like T1D, obesity, atherosclerosis, and IBD, but also against food allergies strongly suggests that we can find a connection between certain kinds of foods that can strengthen the intestinal mucus lining and prevent COVID-19. The mutualistic commensal bacteria can help mitigate clinical conditions like CD, T1D, rheumatoid arthritis, and can also calm down the cytokine storms triggered by COVID-19 and prevent its penetration inside the GI tract. We need to reconsider the importance of GIM in curbing COVID-19 along with other clinical treatments available for COVID-19 [36-41].

\section{Future Prospective}

Can microbiome research teach us how to shape and strengthen our immune system to protect against pathogens and their metabolites? An innovation of a vaccine packed in a dairy product is on the horizon. The spike protein S cloned inside probiotic area pili makes bacteria immunogenic and can be administered as a dairy product (https://tass.com/science/1162979), a yogurt vaccine! Can we administer new commensal microbiomes from other closest mammals, fecal microbiota transplantation, next-generation probiotics like butyrate-producing gut microbes, personalized plant-based medicine to improve our gut health, and strengthen our immune response against COVID-19? Can we add certain types of plants or plant products to enhance immunity against pathogens? I think if we stop deforestation, restore nature, respect and provide space for other living organisms on Earth, and live in harmony with nature, we can not only stop COVID-19 but prevent future pandemics.

\section{Author's Contributions}

\section{KKA wrote and edited the manuscript.}

\section{Acknowledgements}

I am extremely thankful to Dr. Zeng-Jie Yang from Fox Chase Cancer Center for inspiring me to write this article, Dr. Thomas Caceci for sharing the beautiful picture showing salient features of the small and large intestine. Thanks to Ms. Kinu Aneja for editing this article. Thanks to Ms. Mansi Chawla to constantly persuade me to write for ASMI as an editor. Thanks to Indian farmers for sustaining whole planet.

\section{Bibliography}

1. Bäckhed F., et al. "Host-bacterial mutualism in the human intestine”. Science 307.5717 (2005): 1915-1920.

2. Krieg NR., et al. "Phylum XIV. Bacteroidetes phyl. nov." Bergey's Manual of Systematic Bacteriology 4 (2010): 25. Edited by N. R. Krieg, J. T. Staley, D. R. Brown, B. P. Hedlund, B. J. Paster, N. L. Ward, W. Ludwig and W. B.

3. Reichenbach H. "The Order Cytophagales". In: Dworkin M., Falkow S., Rosenberg E., Schleifer KH., Stackebrandt E. [eds] The Prokaryotes (2006). Springer, New York, NY.

4. Moszak M., et al. "You Are What You Eat-The Relationship between Diet, Microbiota, and Metabolic Disorders-A Review". Nutrients 12.4 (2020): 1096.

5. Brown JM and Hazen SL. "The gut microbial endocrine organ: bacterially derived signals driving cardiometabolic diseases". Annual Review of Medicine 66 (2015): 343-359.

6. Clarke G., et al. "Minireview: Gut microbiota: the neglected endocrine organ". Molecular Endocrinology 28.8 (2014): 12211238. 
7. Cullen CM., et al. "Emerging Priorities for Microbiome Research". Frontiers in Microbiology 11 (2020): 136.

8. Liu L and Zhu G. "Gut-brain axis and mood disorder". Frontiers in Psychiatry 9 (2018): 223.

9. Martin C R., et al. "The brain-gut-microbiome axis". Cellular and Molecular Gastroenterology and Hepatology 6 (2018): 133-148.

10. Bäckström M., et al. "Increased Understanding of the Biochemistry and Biosynthesis of MUC2 and Other Gel-Forming Mucins Through the Recombinant Expression of Their Protein Domains". Molecular Biotechnology 54 (2013): 250-256.

11. Fujimoto K and Uematsu S. "Innate Immunity at Mucosal Surfaces". In Mucosal Vaccines, 2nd ed.; Kiyono, H., Pascual, D.W., Eds.; Publisher: London: Elsevier/Academic Press (2020): 101-116.

12. Okumura R and Takeda K. "Roles of intestinal epithelial cells in the maintenance of gut homeostasis". Experimental and Molecular Medicine 49.5 (2017): e338.

13. Vaishnava S., et al. "The antibacterial lectin RegIII-gamma promotes the spatial segregation of microbiota and host in the intestine". Science 334 (2011): 255-258.

14. Johansson ME., et al. "The inner of the two Muc2 mucin-dependent mucus layers in colon is devoid of bacteria". Proceedings of the National Academy of Sciences of the United States of America 105 (2008): 15064-15069.

15. Li G., et al. "Coronavirus infections and immune responses". Journal of Medical Virology 92.4 (2020): 424-432.

16. Li C., et al. "Competitive virus and host RNAs: the interplay of a hidden virus and host interaction". Protein Cell 5 (2014): 348356.

17. Aneja KK., et al. "Can RNAi be used as a weapon against COVID-19/SARSCoV-2?” Microbiology Discovery 8 (2020): 1.

18. Christoph Muus., et al. "Integrated analyses of single-cell atlases reveal age, gender, and smoking status associations with cell type- specific expression of mediators of SARS-CoV-2 viral entry and highlights inflammatory programs in putative target cells". bioRxiv (2020).

19. Catanzaro M., et al. "Immune response in COVID-19: addressing a pharmacological challenge by targeting pathways triggered by SARS-CoV-2". Signal Transduction and Targeted Therapy 5 (2020): 84.
20. Ong J., et al. “COVID-19 in gastroenterology: a clinical perspective”. Gut 69 (2020): 1144-1145.

21. Kim HS. "Do an Altered Gut Microbiota and an Associated Leaky Gut Affect COVID-19 Severity?" mBio 12.1 (2021): e03022-3120.

22. Liu M., et al. "Potential role of ACE2 in coronavirus disease 2019 (COVID-19) prevention and management". Journal of Translational Internal Medicine 8 (2020): 9-19.

23. Zhang Y., et al. "New understanding of the damage of SARSCoV-2 infection outside the respiratory system". Biomedicine and Pharmacotherapy 127 (2020): 110195.

24. Yeoh YK., et al. "Gut microbiota composition reflects disease severity and dysfunctional immune responses in patients with COVID-19". Gut 70.4 (2021): 698-706.

25. Matsuoka K and Kanai T. "The gut microbiota and inflammatory bowel disease". Seminar on Immunopathology 37 (2015): 47-55.

26. Hall AB., et al. "A novel Ruminococcus gnavus clade enriched in inflammatory bowel disease patients". Genome Medicine 9 (2017): 103.

27. Davis-Richardson AG., et al. "Bacteroides dorei dominates gut microbiome prior to autoimmunity in Finnish children at high risk for type 1 diabetes". Frontiers in Microbiology 5 (2014): 678.

28. Schirmer M., et al. "Linking the Human Gut Microbiome to Inflammatory Cytokine Production Capacity". Cell 167.4 (2016): 1125-1136.e8.

29. Lee WJ and Hase K. "Gut microbiota-generated metabolites in animal health and disease". Nature Chemical Biology 10 (2014): 416-424.

30. Thorburn AN., et al. "Diet, metabolites, and "western-lifestyle" inflammatory diseases". Immunity 40 (2014): 833-842.

31. Atarashi K., et al. "Treg induction by a rationally selected mixture of Clostridia strains from the human micro- biota". Nature 50 (2013): 232-236.

32. Smith PM., et al. "The microbial metabolites, short-chain fatty acids, regulate colonic Treg cell homeostasis". Science 341 (2013): 569-573. 
33. Stefka AT., et al. "Commensal bacteria protect against food allergen sensitization". Proceedings of the National Academy of Sciences of the United States of America 111 (2014): 1314513150.

34. Gevers D., et al. "The treatment-naive microbiome in new-onset Crohn's disease". Cell Host and Microbe 15.3 (2014): 382392.

35. Paun A., et al. "Immune recognition and response to the intestinal microbiome in type 1 diabetes". Journal of Autoimmunity 71 (2016): 10-18.

36. Cummings JH and Macfarlane GT. "The control and consequences of bacterial fermentation in the human colon". Journal of Applied Bacteriology 70.6 (1991): 443-459.

37. Markowiak-Kopeć P and Śliżewska K. "The Effect of Probiotics on the Production of Short-Chain Fatty Acids by Human Intestinal Microbiome". Nutrients 12.4 (2020): 1107.

38. Krishnan J., et al. "Toll-like receptor signal transduction". Experimental and Molecular Medicine 39.4 (2007): 421-438.

39. Schuster S., et al. "Antiviral RNAi in Insects and Mammals: Parallels and Differences". Viruses 11.5 (2019): 448.

40. Schlee Martin and Gunther Hartmann. "Discriminating self from non-self in nucleic acid sensing". Nature Reviews Immunology 16.9 (2016): 566.

41. Huang C., et al. "Clinical features of patients infected with 2019 novel coronavirus in Wuhan, China”. Lancet 395 (2020): 497506.

\section{Assets from publication with us}

- Prompt Acknowledgement after receiving the article

- Thorough Double blinded peer review

- Rapid Publication

- Issue of Publication Certificate

- High visibility of your Published work

Website: www.actascientific.com/

Submit Article: www.actascientific.com/submission.php

Emaill us: editor@actascientific.com

Contact us: +919182824667

Citation: Kawalpreet K Aneja. "A Link among Human Gut Microbiome and COVID-19?". Acta Scientific Microbiology 4.5 (2021): $134-141$. 